\title{
Retrieval of an Intrauterine Device Embedded in an Ovary and the Small Intestine
}

\section{Christopher M Tarney* and Bret Guidry}

Department of Obstetrics and Gynecology, Womack Army Medical Center, 2817 Reilly Road, Fort Bragg, NC 28307, USA

\begin{abstract}
The Intrauterine Devices (IUDs) to include the copper T380A IUD and levonorgestrel intrauterine system are effective reversible contraceptives options for women. A well-known but infrequent complication of the IUD is uterine perforation. Most uterine perforations occur at the time of IUD insertion but can also occur spontaneously after placement. IUDs located within the abdomen have potential to cause significant morbidity for patients to include perforation into surrounding structures such as the omentum, mesentery, small intestine, colon, and ovaries. Surgical management should be pursued upon confirmation of uterine perforation prevent sequel. Most misplaced IUDs can be managed by laparoscopic retrieval; however, laparotomy may be required when there is extensive adhesive disease. We present a case of a 28-year-old female who required laparotomy to remove a copper T380A IUD embedded into the small intestine and ovary after perforating through the uterus. The intent of this case report is to illustrate the importance of proper technique when placing IUDs and the steps in retrieving an IUD which is suspected to have perforated the uterus.
\end{abstract}

Keywords: Intrauterine device; Laparoscopy; Perforation; Small intestine; Ovary

\section{Introduction}

The IUDs are forms of Long-Acting Reversible Contraception (LARC) which offer the most effective reversible contraceptive options to females [1]. Additionally, the levonorgestrel intrauterine system has been an effective treatment option for women with abnormal uterine bleeding associated with ovulatory dysfunction [2]. During the 19701980 s the IUDs were frequently used, but now only $5.5 \%$ of women in the United States use this form of birth control [3-5]. Possible cause for the decline in IUD rates originates from litigation surrounding the Dalkon Shield IUD due to concerns with its association with pelvic inflammatory disease and toxic shock syndrome [6]. Nevertheless, the IUDs are a safe and effective contraception with rare complications.

One of the most notable and severe complications of IUDs is uterine perforation. The incidence of uterine perforation is estimated to occur in 1 in 1,000 insertions [7]. The incidence of perforation depends on multiple factors to include anatomic position of the uterus, placement in postpartum period, insertion technique, and operator inexperience [8]. IUDs that have perforated the uterus should be removed surgically by laparoscopy or laparotomy to prevent further complications. Fifteen percent of uterine perforations involve adjacent organs in which possible intestinal ramifications include ischemia, stricture, fistula, perforation, and obstruction [8].

We present a case of laparoscopic evaluation of a copper T380A IUD embedded in the ovary and small intestine that required laparotomy to be removed.

\section{Case}

A 28 year-old, gravid 3, para 2-0-0-2, at unknown gestational age presented to the obstetric clinic after being found to have a quantitative HCG of 32 during a recent emergency room visit for upper respiratory symptoms. Two years prior a copper T380A IUD was placed with successful infertility. The patient reported evaluating for IUD strings each month since placement but this month she was not able to palpate the IUD strings. Bimanual exam revealed an approximately six week sized uterus with extreme retroflexion in which there was moderate tenderness to palpation over the right adnexa with fullness noted on that side. On speculum exam the IUD strings were visualized at the external os. A transvaginal ultrasound showed no intrauterine pregnancy with small amount of fluid in the uterine cavity, trace freefluid in the pelvis, a small ovarian cyst on the left side measuring 1.7 $\mathrm{cm}$, and no IUD within the uterus. A KUB demonstrated the IUD was located in the pelvis.

The patient followed up forty-eight hours later with a repeat quantitative HCG of 15.5. Given that her quantitative HCG demonstrated that this was not a viable pregnancy, attempts were then made to remove the IUD in clinic. A transabdominal ultrasound was used during the attempt which showed a supra-uterine structure adjacent to the IUD that moved when the strings were manipulated. Given these findings in conjunction with patient discomfort, decision was then made to proceed to laparoscopic retrieval of the IUD.

Laparoscopy revealed extensive pelvic adhesions in which the right ovary and loop of small bowel were adherent over the anterior surface of the uterus. The adhesions were taken down with the Harmonic Scalpel which allowed for visualization of a portion of the IUD strings. The strings were then cut and used to grasp for manipulation. The proximal end of the strings originated in the uterine cavity and was exiting the anterior surface of the uterus likely signifying the site of perforation. The distal ends of the strings were tracking into the adhesion of the right ovary and small bowel. These adhesions were then separated with blunt dissection which illustrated one arm of the IUD was embedded into the right ovarian stroma and the other arm of the device appeared to have perforated the small intestine. General surgery was consulted intraoperatively due to concerns for small bowel damage.

*Corresponding author: Christopher M Tarney, Department of Obstetrics and Gynecology, Womack Army Medical Center, 2817 Reilly Road, Fort Bragg, NC 28307, USA, Tel: 908-419-5046; Fax: 910-907-2903; E-mail: christopher.tarney@us.army.mil

Received February 08, 2014; Accepted February 21, 2014; Published February 23, 2014

Citation: Tarney CM, Guidry B (2014) Retrieval of an Intrauterine Device Embedded in an Ovary and the Small Intestine. J Clin Case Rep 4: 347. doi:10.4172/21657920.1000347

Copyright: () 2014 Tarney CM, et al. This is an open-access article distributed under the terms of the Creative Commons Attribution License, which permits unrestricted use, distribution, and reproduction in any medium, provided the original author and source are credited. 
With general surgery assistance, the IUD was removed intact from the ovary and small intestine. The general surgeon recommended converting to laparotomy to further evaluate and repair the small intestine. A Pfannenstiel incision was performed followed by inspection of the bowel. We identified an intramural injury that did not fully perforate into the lumen of the small intestine. This area was repaired by the General Surgeon. There was light bleeding along the ovary which was made hemostatic with cautery. Upon conclusion of the surgery attention was then focused to the vagina where the remaining portions of the IUD strings were removed.

The patient had an uncomplicated postoperative course and was discharged to home on day two after meeting all discharge criteria. She has done well 12 months postoperatively.

\section{Discussion}

The IUDs are one of the most effective long term and reversible forms of contraception, and the levonorgestrel intrauterine system is an effective therapeutic option for women with abnormal uterine bleeding originating from ovulatory dysfunction. The most common adverse effect of the copper T380A IUD is abnormal bleeding and pain [7]. Although uncommon, providers must be cognizant of the potential complication of uterine perforation of an IUD, which typically occurs during placement of the device. Uterine perforations can be complete in which the entire IUD perforates through the uterus or partial in which a portion of the IUD embeds into the myometrium. Most perforations are asymptomatic and go unnoticed at time of placement [9]. Most perforations occur at time of insertion; however, there is speculation that perforations can arise secondarily $[10,11]$.

Prior to placing an IUD, providers need to be aware of certain risk factors that increase the risk for uterine perforation to include an immobile or retroverted uterus, insertion during the postpartum period in which the uterine wall may be thin, and operator inexperience [4]. Providers should be aware of the prescribing information published by the IUD manufacturers with their respective guidelines on techniques and contraindications for placement. A mandatory step that is often overlooked is performing a bimanual exam prior to placement. This necessary step allows one to determine the axis of the uterus and to detect patients who may be at risk for difficult placement. The manufacturers also recommend sounding the uterine cavity prior to placement to determine the size of the cavity. Signs concerning for perforations during placement include significant pelvic pain and placing the device at a distance that exceeds the uterine sound. If a provider is concerned for uterine perforation at time of insertion then one may perform an ultrasound to confirm intrauterine placement of the IUD. This can typically be performed with a transabdominal ultrasound but body habitus may require a transvaginal approach. Providers may consider using an ultrasound upon placement of the IUD in patients who have risk factors for uterine perforation.

Given that perforations may be asymptomatic, clinicians may have patients return to clinic in six to eight weeks after placement to confirm IUD placement. Additionally, patients should be educated on palpating strings on a monthly basis with instructions to schedule follow up if they are unable to palpate the IUD strings. Providers may consider cutting the strings long after placement as they can always be trimmed down at a later date, which mitigates confusion on the presence or absence of strings.

Patients with a perforated IUD may present with varying symptoms to include pelvic pain, abnormal vaginal bleeding, inability to palpate IUD strings, and a positive pregnancy test. If there is concern for possible uterine perforation of an IUD then further diagnostic evaluation is warranted to prevent complications. One of the first steps is to visualize the IUD strings exiting the cervical os on speculum exam. An important point to emphasize is that visualization of the IUD strings exiting the cervical os does not rule out partial or complete uterine perforation. Ultrasound should then be performed to determine if the IUD is within the uterine cavity. If there is no evidence of the IUD within the uterus then an X-Ray can be performed to evaluate whether the IUD is in the pelvis. One may consider imaging with computed tomography if there is concern for involvement or injury to surrounding organs.

Most uterine perforations of an IUD are uncomplicated [10]. However, once perforation of the uterus is confirmed then surgical removal is required to prevent injury to surrounding structures. Misplaced IUDs may lead to long term pain, adhesions, and penetration into surrounding structures such as the bladder, colon, appendix, and small intestine, and vessels $[9,12]$. Perforated IUDs may be removed electively with laparoscopic surgery; however, laparotomy may be required for patients who have extensive adhesive disease [13]. Bowel perforation can lead to abscess formation, volvulus, and intestinal ischemia which can become life-threatening for the patient $[9,10]$.

To our knowledge, this is the first case presented of a copper T380A IUD embedded in the ovary and small intestine after a perforation of the uterus. Given the severe angle of retroflexion of the uterus on bimanual exam, we postulate that the uterine perforation had likely occurred during placement of the IUD, which also explains the extensive adhesions surrounding the IUD.

\section{Conclusion}

The IUDs remain an important, safe, an effective form of long acting reversible contraception. Although infrequent, uterine perforation is a well-recognized complication of these devices. Providers should be aware of risk factors associated with uterine perforation and should take appropriate steps in carefully placing these devices to prevent perforation. If uterine perforation is confirmed then appropriate surgical management either via laparoscopy or laparotomy should immediately be undertaken to prevent long-term complications.

\section{Disclaimer}

The opinions or assertions contained herein are the private views of the authors and are not to be construed as the official policy of the Department of the Army, Department of Defense, or the U.S. Government.

\section{References}

1. American College of Obstetricians and Gynecologists (2011) ACOG Practice Bulletin No. 121: Long-acting reversible contraception: Implants and intrauterine devices. Obstet Gynecol 118: 184-196.

2. Committee on Practice Bulletinsâ€"Gynecology (2013) Practice bulletin no. 136: management of abnormal uterine bleeding associated with ovulatory dysfunction. Obstet Gynecol 122: 176-185.

3. Mosher WD, Jones J (2010) Use of contraception in the United States: 1982 2008. Vital Health Stat 23: 1-44.

4. Andersson K, Ryde-Blomqvist E, Lindell K, Odlind V, Milsom I (1998) Perforations with intrauterine devices. Report from a Swedish survey. Contraception 57: 251-255.

5. Fortney JA, Feldblum PJ, Raymond EG (1999) Intrauterine devices. The optimal long-term contraceptive method? J Reprod Med 44: 269-274.

6. Mishell DR Jr (1998) Intrauterine devices: mechanisms of action, safety, and efficacy. Contraception 58: 45S-53S. 
Citation: Tarney CM, Guidry B (2014) Retrieval of an Intrauterine Device Embedded in an Ovary and the Small Intestine. J Clin Case Rep 4: 347. doi:10.4172/2165-7920.1000347

7. Brockmeyer A, Kishen M, Webb A (2008) Experience of IUD/IUS insertions and clinical performance in nulliparous women--a pilot study. Eur J Contracept Reprod Health Care 13: 248-254

8. Arslan A, Kanat-Pektas M, Yesilyurt H, Bilge U (2009) Colon penetration by a copper intrauterine device: a case report with literature review. Arch Gynecol Obstet 279: 395-397

9. Mederos R, Humaran L, Minervini D (2008) Surgical removal of an intrauterine device perforating the sigmoid colon: a case report. Int J Surg 6: e60-62.

10. Zakin D, Stern WZ, Rosenblant R (1981) Complete and partial uterine perforation and embedding following insertion of intrauterine devices: I.
Classification, complications, mechanism, incidence, and missing string. Obstet Gynecol Survey 36: 335-353.

11. Thomalla JV (1986) Perforation of urinary bladder by intrauterine device Urology 27: 260-264.

12. Chi E, Rosenfeld D, Sokol TP (2005) Laparoscopic removal of an intrauterine device perforating the sigmoid colon: a case report and review of the literature. Am Surg 71: 1055-1057.

13. Ozgun MT, Batukan C, Serin IS, Ozcelik B, Basbug M, et al. (2007) Surgical management of intra-abdominal mislocated intrauterine devices. Contraception 75: 96-100. 